\title{
Caries dentales en pacientes pediátricos durante el período de encierro del COVID-19
}

\section{Dental caries in pediatric patients during the COVID-19 lockdown period}

\author{
Marcos Roberto Tovani-Palone ${ }^{1}$, Valentino Natoli²] \\ ${ }^{1}$ Universidade de São Paulo, Faculdade de Medicina de Ribeirão Preto. Ribeirão Preto, Brasil. \\ ${ }^{2}$ European University of Madrid, School of Biomedical and Health Sciences, Department of Dentistry. \\ Madrid, Spain.
}

La enfermedad del coronavirus 2019 (COVID-19) es una enfermedad sistémica ${ }^{(1-3)}$, que ha provocado la muerte de miles de personas en todo el mundo, afectando incluso a los países de América Latina ${ }^{(4-6)}$. Desde que fue declarada pandemia, la enfermedad COVID-19 ha sido un gran desafío para muchos sectores. En este contexto, el colapso de los sistemas de salud en diferentes países ha sido frecuente, incluso en naciones desarrolladas, dada la necesidad de disponibilidad de un gran número de camas ambulatorias y hospitalarias, así como de equipos altamente especializados para el tratamiento de $\operatorname{casos} \operatorname{severos}^{(7,8)}$.

En un intento por contener la propagación del virus causante de la COVID-19, denominado coronavirus del síndrome respiratorio agudo severo 2 (SARSCoV-2), las autoridades sanitarias y los gobiernos han promulgado drásticas medidas de contención. Esto, a su vez, ha tenido diferentes impactos en la vida cotidiana de las poblaciones $^{(9-1)}$.

También vale la pena mencionar aquí que el suministro y la continuidad de los tratamientos dentales se han visto muy afectados. Una de las principales causas de esto debe estar relacionada con los riesgos de infección por SARS-CoV-2 en el medio dental, principalmente por el aerosol generado durante los procedimientos. Además, durante los períodos de encierro ha sido necesario interrumpir las actividades electivas de rutina en el campo odontológico, razón por la cual muchos pacientes vieron interrumpidos sus planes de tratamiento, ya sean preventivos o curativos. En general, solo se ha brindado atención de emergencia durante estos periodos $^{(12)}$.

Además, en un estudio reciente publicado por Ortellado et al. (2021) se mostró que los niños presentan cambios en los hábitos alimentarios durante el período de encierro del COVID-19 y en general tienden a tener una ingesta de alimentos más frecuente y rica en azúcares ${ }^{(13)}$, lo que se traduce en mayores riesgos de caries dental y de otros problemas bucales graves, como los abscesos dentales. Por lo tanto, dado que la caries dental es una disbiosis ${ }^{(14)}$, es de gran valor implementar acciones dirigidas a orientar a padres e hijos sobre la importancia de hábitos alimentarios más saludables, así como sobre la realización de una higiene bucal adecuada y segura durante este período. El entorno escolar virtual debe ser un medio propicio para ese fin.

Recomendaciones esenciales para mantener la higiene bucal pediátrica durante los períodos de encierro:

Correspondencia: Marcos Roberto Tovani-Palone.Correo: marcos_palone@hotmail.com Recibido: 5/07/2021 Aceptado: 18/10/2021 DOI: https://doi.org/10.31698/ped.48032021002

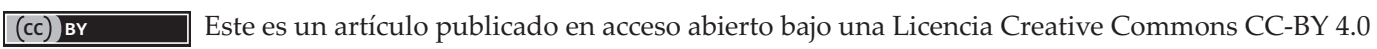


- Realice la higiene bucal al menos 3 veces al día, especialmente antes de acostarse

- Use una cantidad apropiada de pasta de dientes con flúor

- Siempre use hilo dental

- Los cepillos de dientes deben ser de cabeza pequeña y con cercas suaves o extra suaves

- El tubo dental debe usarse individualmente (para evitar el riesgo de infección por SARS-CoV-2)

- Mantenga el cepillo de dientes de cada miembro de la familia por separado (para evitar el riesgo de infección por SARS-CoV-2)

- Disminuir la frecuencia de ingesta de alimentos ricos en azúcar a lo largo del día

COVID-19 seguirá teniendo un gran impacto sobre la práctica de la odontología pediátrica. Sin embargo, podemos impulsar cambios en algunos de

\section{REFERENCIAS}

1. Khawaja UA, Shamsoddin E, Desideri LF, TovaniPalone MR. Infection of red blood cells by SARS-CoV-2: new evidence. einstein (São Paulo). 2021; 19:eCE6285. doi: https://doi.org/10.31744/einstein_journal/2021CE6285

2. Giacalone M, Tovani-Palone MR, Marin L, Febbi M, Russano T, Giacalone A. Neurological and neuropsychiatric disorders associated with COVID-19 Part I: overview and neurological disorders. einstein (São Paulo). 2021;19:eCE6448. doi: https://doi.org/10.31744/ein stein_journal/2021CE6448

3. Giacalone M, Tovani-Palone MR, Marin L, Febbi M, Russano T, Giacalone A. Neurological and neuropsychiatric disorders associated with COVID-19 Part II: neuropsychiatric disorders and final consideration. einstein (São Paulo). 2021;19:eCE6464. doi: https://doi.org/10.31744/einstein_journal/2021CE6464

4. Johns Hopkins Coronavirus Resource Center. World Map [Internet]. Baltimore [accessed 2021 May 14]. Available at: https://coronavirus.jhu.edu/map.html

5. Mejia CR, Charri JC, Rodriguez-Alarcon JF, FloresLovon K, Cuzcano-Gonzales KV, Benites-Ibarra CA, et al. Perception of possible SARS-CoV-2 infection and associated complications in seven labor sectors in Peru. Electron J Gen Med. 2021 ;18(2):em279. doi: https://doi. org/10.29333/ejgm/9730 los enfoques de la odontología pediátrica utilizando prácticas más centradas en la prevención, principalmente con el objetivo de motivar a los padres y pacientes pediátricos a adoptar mejores hábitos para interceptar y reducir el desarrollo de la caries dental.

\section{CONTRIBUCIONES DE AUTORÍA}

\section{Marcos Roberto Tovani-Palone}

Ha participado en la concepción del artículo, redacción del artículo, supervisión, revisión y aprobación de la versión final.

\section{Valentino Natoli}

Ha participado en la redacción del artículo, revisión y aprobación de la versión final.

6. Mejia CR, Rodriguez-Alarcon JF, Vera-Gonzales JJ, Ponce-Lopez VL, Chamorro-Espinoza SE, Quispe-Sancho A, et al. Fear perception of the COVID-19 pandemic in Peru. Electron J Gen Med. 2021; 18(3):em285. doi: https://doi.org/10.29333/ejgm/9764

7. Pedersini P, Villafañe JH, Corbellini C, Tovani-Palone MR. COVID-19 pandemic: a physiotherapy update. Electron J Gen Med. 2021; 18(1):em264. doi: https://doi. org/10.29333/ejgm/8574

8. Tovani-Palone MR, Shamsoddin E. Use of mouthwashes in the management of COVID-19 patients in intensive care units: recommendations and current evidence. einstein (São Paulo). 2021;19:eCE6419. doi: https://doi.org/10.31 744/einstein_journal/2021CE6419

9. Nafees M, Khan F. Pakistan's response to COVID-19 pandemic and efficacy of quarantine and partial lockdown: a review. Electron J Gen Med. 2020; 17(6):em240. doi: https://doi.org/10.29333/ejgm/7951

10. Desideri LF, Tovani-Palone MR. COVID-19 and the increased risk of myopia and digital eye strain. einstein (São Paulo). 2021; 19:eCE6491. doi: https://doi.org/10.31 744/einstein_journal/2021CE6491 
11. Ferro Desideri L, Barra F, Tovani-Palone MR. COVID19 and refractive errors: a new potential threat for children and adolescents. Electron J Gen Med. 2021; 18(4):em300. doi: https://doi.org/10.29333/ejgm/10870

12. Shamsoddin E, DeTora LM, Tovani-Palone MR, Bierer BE. Dental care in times of the COVID-19 pandemic: a review. Med Sci (Basel). 2021; 9(1):13. doi: https://doi. org/10.3390/medsci9010013
13. Sotomayor Ortellado R, Matiauda Otaño A, Ferreira Cabañas A, Canese Krivoshein A. Dieta, higiene bucal y riesgo de caries dental en niños escolares de Concepcion, durante el confinamiento por COVID-19. Pediatr. (Asunción). 2021; 48(1): 65-72. doi: https://doi.org/10.31 698/ped.48012021011

14. Tanner ACR, Kressirer CA, Rothmiller S, Johansson I, Chalmers NI. The caries microbiome: implications for reversing dysbiosis. Adv Dent Res. 2018;29(1):78-85. doi: https://doi.org/10.1177/0022034517736496 